\title{
Carbonaceous Material in Extra-terrestrial Matter
}

\author{
Zita Martins \\ Dept of Earth Science and Engineering, Imperial College London, South Kensington Campus, \\ London SW7 2AZ, UK \\ email: z.martins@imperial.ac.uk
}

\begin{abstract}
Comets, asteroids, meteorites, micrometeorites, interplanetary dust particles (IDPs), and ultra-carbonaceous Antarctic micrometeorites (UCAMMs) may contain carbonaceous material, which was exogenously delivered to the early Earth. Carbonaceous chondrites have an enormous variety of extra-terrestrial compounds, including all the key compounds important in terrestrial biochemistry. Comets contain several carbon-rich species and, in addition, the hypervelocity impact-shock of a comet can produce several $\alpha$-amino acids. The analysis of the carbonaceous content of extra-terrestrial matter provides a window into the resources delivered to the early Earth, which may have been used by the first living organisms.
\end{abstract}

Keywords. meteors, meteoroids

\section{Introduction}

The inner solar system was bombarded from around 4.5 to 3.8 billion years ago (Schidlowski 1988; Schopf 1993; Chyba and Sagan 1992). These impacts may be observed by the impact craters on planetary bodies such as the Moon. Comets, asteroids and their fragments (i.e. meteorite, micrometeorites and interplanetary dust particles (IDPs)) are known to contain carbonaceous material, which was exogenously delivered to the early Earth during that period of time (Anders 1989; Chyba et al. 1990; Chyba and Sagan 1992). Interplanetary dust particles (IDPs) can contain up to $50 \%$ of organic carbon by mass, but typically have $10 \%$ organic carbon by mass (Anders 1989; Schramm et al. 1989). Micrometeorites and IDPs are known to contain organic molecules, such as polycyclic aromatic hydrocarbons (PAHs) and alkylated derivatives, aliphatic hydrocarbons, ketones and amino acids (Clemett et al. 1993; Matrajt et al. 2004, 2005). Furthermore, some aromatic compounds present in IDPs are associated with D/H anomalies (Wopenka 1988), while the long aliphatic chains with little ramifications present in IDPs may have originated at the edges of the protoplanetary disk (Matrajt et al. 2013). However, it is not certain whether all these molecules, in particular the amino acids are indeed indigenous or terrestrial contamination (Clemett et al. 1993; Brinton et al. 1998; Flynn 2003; Matrajt et al. 2004). Ultra-carbonaceous Antarctic micrometeorites (UCAMMs) contain up to $90 \%$ of carbonaceous material (Duprat et al. 2010; Dartois et al. 2013). UCAMMs contain unusually high nitrogen- and deuterium-rich organic matter, with bulk atomic $\mathrm{N} / \mathrm{C}$ ratios of 0.05 and 0.12 (locally exceeding 0.15 ). They were proposed to be formed in very low temperature regions of the Solar System, such as the surface of small objects beyond the trans-neptunian region (Dartois et al. 2013). Carbonaceous meteorites contain up to $5 \mathrm{wt} \%$ of organic carbon (Alexander et al. 2013), which is either locked in an insoluble kerogen-like polymer, or in a rich organic inventory of soluble organic compounds (Cronin and Chang 1993; Cody and Alexander 2005; Martins and Sephton 2009). There are several differences in abundance and distribution of the soluble organic 
content between different carbonaceous meteorites. This may result from different physical and chemical processes such as thermal metamorphism (Burton et al. 2011; Chan et al. 2012), or aqueous alteration on the meteorite parent body (Glavin et al. 2006; Martins et al. 2015). Comets also have several extra-terrestrial organic molecules (Crovisier and Bockelée-Morvan 1999; Ehrenfreund et al. 2002), including the simplest amino acid glycine (Elsila et al. 2009). Most recently the results from the Rosetta mission of the surface of comet $67 \mathrm{P} /$ Churyumov-Gerasimenko were published. Carbon-rich species, including alcohols, carbonyls, amines, nitriles, amides, isocyanates, and the radiation-induced polymer polyoxymethylene were detected (Goesmann et al. 2015; Wright et al. 2015). In addition, the impact-shock of comets produces complex organic molecules (Martins et al. 2013).

\section{Synthesis of amino acids via the impact-shock of comets}

Goldman et al. (2010) performed ab initio molecular dynamics simulations that showed that shock waves passed into ice mixtures representative of comets, could theoretically yield amino acids. Amino acid precursors (such as ammonia, methanol and carbonyl compounds) have been observed in comets, e.g. Halley, Hyakutake, Tempel 1, GiacobiniZinner, Hartley 2 and Hale-Bopp (Festou et al. 2005; DiSanti et al. 2013; Crovisier and Bockelée-Morvan 1999; Ehrenfreund et al. 2002; Ehrenfreund and Charnley 2000; Mumma et al. 2003; Bockelée-Morvan et al. 2000). Therefore, it has been experimentally tested in the laboratory whether the hypervelocity impact-shock of a typical comet ice mixture would synthesize amino acids (Martins et al. 2013). Results show that the impacts of comets onto rocky surfaces, and the impacts of meteorites onto icy surfaces (such some of the Jovian and Saturnian satellites) produce several $\alpha$-amino acids. Several lines of evidence prove that the detected amino acids were indeed synthesized by impact-shock (Martins et al. 2013):

- A racemic mixture of alanine was detected, with a $\mathrm{D} / \mathrm{L}$ ratio of 0.99 , and a racemic mixture of norvaline was also detected with a $\mathrm{D} / \mathrm{L}$ ratio of 0.97 ;

- Structural diversity and the decrease of amino acid abundances with increasing number of carbon atoms were observed;

- The non-protein amino acids $\alpha$-aminoisobutyric acid ( $\alpha$-AIB) and isovaline were obtained;

- The synthesis is in agreement with ab initio molecular dynamics simulations modelling (Goldman et al. 2010);

- The control ice samples and the extraction procedural blanks were completely free from organic compounds.

The synthetic pathway to create amino acids via impact-shock is not completely clear. Suggested synthetic pathway includes the Strecker-cyanohydrin synthesis using $\alpha$-amino acid precursors (carbonyl compounds, ammonia, and hydrogen cyanide). In this case, the carbonyl precursors (aldehydes and ketones) would be synthesised by the oxidation of methanol (present in the simulated ice mixture), which would generate the $\alpha$-amino acid precursor carbonyl compounds. Support for this is indicated by an ice mixture containing only ammonia and carbon dioxide and lacking methanol. Even with an impact velocity of $7.12 \mathrm{~km} / \mathrm{s}$, no detectable quantities of amino acids were produced (Martins et al. 2013). To note, however that the impact shock occurs on timescales of nanoseconds to milliseconds, which should not be consistent with the Strecker-cyanohydrin synthesis. Alternatively, a high shock pressure (i.e. at $7 \mathrm{~km} / \mathrm{s}$ the peak shock pressure experienced by the ice mixture is $50 \mathrm{GPa}$ (Martins et al. 2013; Goldman et al. 2010)) results in the 
formation of ions and radicals, which will then be involved in the post-shock reaction to form amino acids.

In summary, the exogenous delivery of carbonaceous material in our Solar System expands the inventory of locations where life could have potentially originated. In fact, the detection by future space missions of an amino acid racemic ratio on the surface of icy Jovian and Saturnian satellites may be the result of impact shock events as well as the contribution from meteorites.

\section{References}

Alexander, C. M. O.'D., Howard, K. T., Bowden, R., \& Fogel, M. L. 2013, GCA, 123, 244-260 . Anders, E. 1989, Nature, 342, 255-257.

Bockelée-Morvan, D., Lis, D. C., Wink, J. E., Despois, D., Crovisier, J., Bachiller, R., Benford, D. J., Biver, N., Colom, P., Davies, J. K., Gérard, E., Germain, B., Houde, M., Mehringer, D., Moreno, R., Paubert, G., Phillips, T. G., \& Rauer, H. 2000, A\&A, 353, 1101-1114.

Brinton, K. L. F., Engrand, C., Glavin, D. P., Bada, J. L., \& Maurette, M. 1998. OLEB, 28, 413-424.

Burton, A. S., Glavin, D. P., Callahan, M. P., Dworkin, J. P., Jenniskens, P., \& Shaddad, M. H. 2011, Meteoritics \& Planetary Science, 46, 1703-1712.

Chan, H.-S., Martins, Z., \& Sephton, M. A. 2012. Meteoritics and Planetary Science, 47, 15021516.

Chyba, C. F. \& Sagan, C. 1992, Nature, 355, 125-132.

Chyba, C. F., Thomas, P. J., Brookshaw, L., \& Sagan, C. 1990, Science, 249, 366-373.

Clemett, S. J., Maechling, C. R., Zare, R. N., Swan, P. D., \& Walker, R. M. 1993. Science, 262, 721-725.

Cody, G. D. \& Alexander, C. M. O.'D. 2005, Geochimica et Cosmochimica Acta, 69, 1085-1097.

Cronin, J. R. \& Chang, S. 1993, In The Chemistry of Life's Origin, edited by Greenberg J. M., Mendoza-Gomez C. X. and Pirronello V. Dordrecht: Kluwer. pp. 209-258.

Crovisier, J. \& Bockelee-Morvan, D. 1999, Space Science Reviews, 90, 19-32.

Dartois, E., Engrand, C., Brunetto, R., Duprat, J., Pino, T., Quirico, E., Remusat, L., Bardin, N., Briani, G., Mostefaoui, S., Morinaud, G., Crane, B., Szwec, N., Delauche, L., Jamme, F., Sandt, Ch., \& Dumas, P. 2013, Icarus, 224, 243-252.

DiSanti M. A., Bonev, B. P., Villanueva, G. L., \& Mumma M. J. 2013, The Astrophysical Journal, $763,1$.

Duprat, J., Dobrica, E., Engrand, C., Aléon, J., Marrocchi, Y., Mostefaoui, S., Meibom, A., Leroux, H., Rouzaud, J.-N., Gounelle, M., \& Robert, F. 2010, Science, 328, 742-745.

Ehrenfreund, P., Irvine, W., Becker, L., Blank, J., Brucato, J. R., Colangeli, L., Derenne, S., Despois, D., Dutrey, A., Fraaije, H., Lazcano, A., Owen, T., \& Robert, F., International Space Science Institute ISSI-Team 2002, Reports on Progress in Physics, 65, 1427-1487.

Ehrenfreund, P. \& Charnley, S. B. 2000, Annual Review of Astronomy and Astrophysics, 38, 427-483.

Elsila, J. E., Glavin, D. P., \& Dworkin, J. P. 2009, Meteoritics and Planetary Science, 44, 1323.

Festou M., Uwe-Keller, H. \& Weaver, H. A. 2005, Comets-I. I. University of Arizona Press. Flynn, G. J., Keller, L. P., Feser, M., Wirick, S., \& Jacobsen, C. 2003, GCA, 67, 4791-4806.

Glavin, D. P., Dworkin, J. P., Aubrey, A., Botta, O., Doty, J. H., Martins, Z., \& Bada, J. L. 2006, Meteoritics \& Planetary Science, 41, 889-902.

Goesmann, F. et al. 2015, Science, 349, aab0689-1- aab0689-3.

Goldman, N., Reed, E. J., Fried, L. E.; William Kuo, I.-F., \& Maiti, A. 2010, Nature Chemistry, 2, 949-954.

Matrajt, G., Pizzarello, S., Taylor, S., \& Brownlee, D. 2004, Meteoritics $\&$ Planetary Science, 39, 1849-1858.

Matrajt, G., Muñoz Caro, G. M., Dartois, E., D’Hendecourt, L., Deboffle, D., \& Borg, J. 2005, A $\mathscr{E} A, 433,979-995$.

Matrajt, G., Flynn, G., Brownlee, D., Joswiak, D., \& Bajt, S. 2013, ApJ, 765, 145, 18 pp. Martins, 
Z. M. \& Sephton, A. 2009, In Hughes, A.B. (Ed.), Amino acids, peptides and proteins in organic chemistry. Wiley-VCH Verlag GmbH \& Co. KGaA, Weinheim, pp. 3-42.

Martins, Z., Price, M. C., Goldman, N., Sephton, M. A., \& Burchell, M. J. 2013, Nature Geoscience, 6, 1045-1049.

Martins, Z., Modica, P., Zanda, B., \& Le Sergeant D'Hendecourt, L. 2015, Meteoritics ES Planetary Science, 50, 926-943.

Mumma, M. J., DiSanti, M. A., Dello Russo, N., Magee-Sauer, K., Gibb, E., \& Novak, R. 2003, AdSR, 31, 2563-2575.

Schidlowski, M. 1988, Nature, 333, 313-318.

Schopf, J. W. 1993, Science, 260, 640-646.

Schramm, L. S., Brownlee, D. E., \& Wheelock, M. M. 1989. Meteoritics, 24, 99-112.

Wopenka, B. 1988, EPSL, 88, 221-231.

Wright, I. P., Sheridan, S., Barber, S. J., Morgan, G. H., Andrews, D. J., \& Morse, A. D. 2015, Science, 349, aab0673-1-aab0673-3. 